\title{
Thresholds of Lasing and Modal Patterns of a Limacon Cavity Analysed with Muller's Integral Equations
}

\author{
E. I. Smotrova, Member, IEEE and A. I. Nosich, Fellow, IEEE
}

\author{
Institute of Radiophysics and Electronics of National Academy of Sciences of Ukraine, Kharkov, Ukraine
}

\begin{abstract}
We consider the lasing characteristics of the natural modes in a limacon active microcavity. The modes are the solutions to the two-dimensional (2-D) linear eigenproblem for the Maxwell equations with exact boundary conditions and radiation condition at infinity. This problem is reduced equivalently to the set of Muller's integral equations of the Fredholm second kind and discretized using the exponentially convergent quadrature formulas. The numerical studies of lasing thresholds and modal field patterns of the whispering-gallery modes are presented. They demonstrate that the directionality of emission from a cavity deformed from the circle can be enhanced in several times using relatively small deformations. However this is achieved at the expense of higher threshold values.
\end{abstract}

\section{INTRODUCTION}

Circular microdisk lasers work on the so-called whisperinggallery (WG) modes whose fields experience almost total internal reflection along the rim of the disk. This leads to ultralow material thresholds of lasing for these modes when the disks are pumped. However the circular shape of the cavity also entails a low directionality of light emission. Indeed, any WG mode with the azimuth index $m$ has $2 m$ identical beams in the disk plane emitting the light to infinity. Directionality can be conveniently quantified with the aid of directivity as a ratio of the power emitted into the main beam direction to the total power averaged over all directions. Then any mode of a circular cavity with $m>0$ has directivity equal to 2 .

Thus, it is evident that low threshold and high directivity are, generally speaking, two contradictory demands. Any improvement of the directionality needs a distortion of the cavity shape from the circle that inevitably results in a threshold higher than for the circle. Only a small deformation of circular symmetry may enable one to make the threshold growth of quasi-WG modes tolerable.

Here, non-circular cavities with only one symmetry line can be considered as the best candidates. This is because, from the one hand, they are able to provide a well-shaped main beam of emission and this beam is destined to radiate along the symmetry line. Thus, its angle of emission is fixed due to the symmetry, unlike a spiral cavity mode where it may take an arbitrary and a priori unknown value. A limacon cavity is an example of a slightly deformed circular shape with one symmetry line. Recently, it has been studied theoretically [1-3] and experimentally [2-4] and the improvement of directionality has been confirmed. Still there is no information available on the change of the lasing thresholds in a limacon-deformed microcavity laser. Therefore in this paper we report on the preliminary results of the "warm-cavity" modelling of the lasing modes characteristics in a uniformly active 2-D limacon laser model.

\section{EIgenVAlue Problem for Active MicrocaVities}

Denote the interior domain of a 2-D model of an active dielectric (non-magnetic) microcavity as $D_{i}$, its closed contour as $\Gamma$, and the outer domain as $D_{e}$. Consider a function $U(x, y)$, which is either the $E_{z}$ or the $H_{z}$ field component. When simulating a microlaser, we are interested in the realvalued pairs of numbers $(k, \gamma)$ generating non-zero functions $U$ that solve, off $\Gamma$, the Helmholtz equation $\left(\Delta+k^{2} v^{2}\right) U=0$ with a piecewise-constant refractive index $v$ equal to $v_{i}=\alpha_{i}-i \gamma \quad(\gamma>0)$ in $D_{i}$, and $v_{e}=\alpha_{e}$ in $D_{e}$. Here, the following two-side boundary conditions are imposed on $\Gamma$ : $U^{e}=U^{i}$ and $\eta_{e} \partial U^{e} / \partial n=\eta_{i} \partial U^{i} / \partial n$, where the superscripts " $i, e$ " refer to the corresponding domains, $\eta_{i, e}=1 \quad$ (Epolarisation) or $\eta_{i, e}=1 / v_{i, e}^{2}$ (H-polarisation), and $\vec{n}$ is the outward normal vector to $\Gamma$. Furthermore, the time-averaged electromagnetic energy must be locally integrable to prevent source-like field singularities, and the Sommerfeld radiation condition must be satisfied at infinity. Note that in the 2-D models of thin-cavity lasers the refractive index is understood as the effective refractive index, which is somewhat smaller than the bulk refractive index of the cavity material [5]. The formulated above problem is the Lasing Eigenvalue Problem (LEP), and frequencies $k$ and threshold gains $\gamma$ are the LEP eigenpairs. The essentials and the general theory of LEP approach can be found in [6].

For the contour $\Gamma$ representation, we use the following analytic $2 \pi$-periodic function, where $t \in[0,2 \pi]$ :

$$
x(t)=a(1+\delta \cos t) \cos t, \quad y(t)=a(1+\delta \cos t) \sin t
$$


The curve (1) is called a limacon. Here, $\delta$ is the normalized contour shape parameter, so that if $\delta=0$ then (1) turns to a circle. If, however, $\delta$ take large values, then (1) obtains a characteristic snail-like shape ("limacon" is snail in French). Note that the values of $\delta \leq 0.5$ provide convex contours. For the larger values of $\delta<1$ we have a contour with a dimple, and for $\delta>1$ we obtain a contour with a self crossing.

\section{MULLER’S INTEGRAL EQUATIONS}

Introduce the Green's functions $G_{j}(R)=(i / 4) H_{0}^{(1)}\left(k v_{j} R\right)$ of the homogeneous media, where $j=i, e, R=\left|\vec{r}-\vec{r}^{\prime}\right|$ is the distance between the points $\vec{r}$ and $\vec{r}^{\prime}$, and $H_{0}^{(1)}(\cdot)$ is the Hankel function. After applying the second Green's formula to the functions $G_{j}\left(\vec{r}, \vec{r}^{\prime}\right)$ and $U_{j}$, using boundary conditions, and taking into account the properties of single-layer and double layer potentials, we obtain two integral equations as

$$
\begin{aligned}
& \varphi(\vec{r})+\int_{\Gamma} \varphi\left(\vec{r}^{\prime}\right) A\left(\vec{r}, \vec{r}^{\prime}\right) d l^{\prime}-\int_{\Gamma} \psi\left(\vec{r}^{\prime}\right) B\left(\vec{r}, \vec{r}^{\prime}\right) d l^{\prime}=0, \\
& \frac{\eta_{i}+\eta_{e}}{2 \eta_{e}} \psi(\vec{r})+\int_{\Gamma} \varphi\left(\vec{r}^{\prime}\right) C\left(\vec{r}, \vec{r}^{\prime}\right) d l^{\prime}-\int_{\Gamma} \psi\left(\vec{r}^{\prime}\right) D\left(\vec{r}, \vec{r}^{\prime}\right) d l^{\prime}=0,
\end{aligned}
$$

where $d l^{\prime}$ is the element of the arc on $\Gamma, \varphi(\vec{r})=U_{i}(\vec{r})$ and $\psi(\vec{r})=\partial U_{i}(\vec{r}) / \partial n, \quad \vec{r} \in \Gamma$.

Here, the kernel functions are

$$
\begin{gathered}
A\left(\vec{r}, \vec{r}^{\prime}\right)=\partial G_{i}\left(\vec{r}, \vec{r}^{\prime}\right) / \partial n^{\prime}-\partial G_{e}\left(\vec{r}, \vec{r}^{\prime}\right) / \partial n^{\prime}, \\
B\left(\vec{r}, \vec{r}^{\prime}\right)=G_{i}\left(\vec{r}, \vec{r}^{\prime}\right)-\eta_{i} / \eta_{e} G_{e}\left(\vec{r}, \vec{r}^{\prime}\right) \\
C\left(\vec{r}, \vec{r}^{\prime}\right)=\partial^{2} G_{i}\left(\vec{r}, \vec{r}^{\prime}\right) / \partial n \partial n^{\prime}-\partial^{2} G_{e}\left(\vec{r}, \vec{r}^{\prime}\right) / \partial n \partial n^{\prime}, \\
D\left(\vec{r}, \vec{r}^{\prime}\right)=\partial G_{i}\left(\vec{r}, \vec{r}^{\prime}\right) / \partial n-\left(\eta_{i} / \eta_{e}\right) \partial G_{e}\left(\vec{r}, \vec{r}^{\prime}\right) / \partial n
\end{gathered}
$$

Note that the kernel functions $A(t, \tau)$ and $D(t, \tau)$ are continuous, and the kernel functions $B(t, \tau)$ and $C(t, \tau)$ have logarithmic singularities.

\section{DISCRETISATION OF INTEGRAL EQUATIONS}

One of the most efficient discretisation techniques is the method of quadratures, also known as the Nystrom method [7][10]. This latter method is based on the replacement of the integrals with approximate sums using the appropriate quadrature formulas. As some of the kernel functions have logarithmic singularities, it is convenient to represent all of the kernels in (3) and (4) in such a way that these singularities are extracted [8], [9]. Then the integrals are approximated by two different quadrature rules for the regular and singular parts with the same equidistant set of points. Namely, we use a trigonometric quadrature rule for the parts with logarithmic singularities and a trapezoidal rule for the regular parts [10]. By evaluating the integrals from (2) with the aid of the quadrature rules, we obtain a determinantal equation for the eigenvalues. A secant-type iterative method [1] is further used to find the eigenvalues numerically as roots of this equation.

\section{NUMERICAL RESULTS}

In this paper we consider the convex limacon contour as a continuous deformation of the circle, and therefore denote the modes of the deformed cavity using the notations of their limiting forms in the circle, with a prefix "quasi". Note also that the deformation from the circular symmetry leads to the removal of the double mode degeneracy, for all modes with azimuth indices $m>0$. This is quite similar to our previous study of the modes in a spiral cavity laser [10] and a more recent simulation of a kite-shaped laser [11]. As far as limacon cavity has one symmetry line, the modes break up into two classes according to the symmetry or the anti-symmetry properties of modal fields. Notations "even" for the former and "odd" for the latter modes will reflect this feature.

Near-field patterns of two modes of the doublet $\mathrm{WGH}_{9,1}$ are presented in Fig. 2.

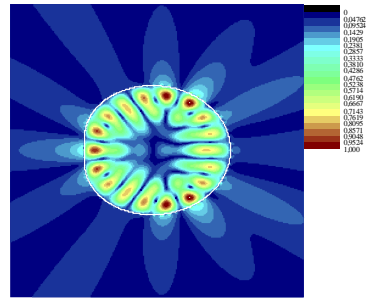

(a)

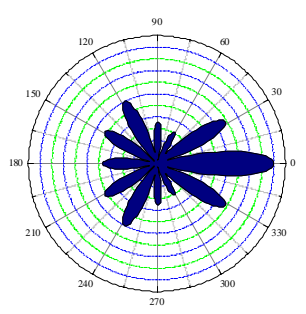

(c)

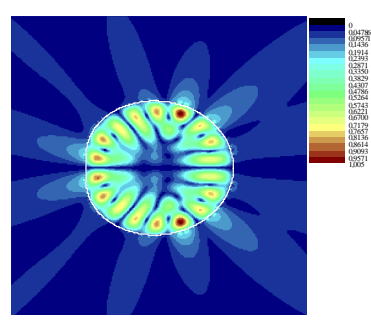

(b)

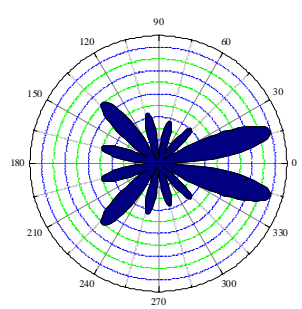

(d)
Fig. 2. Normalized near- and far-field patterns for the doublet of quasi- $\mathrm{WGH}_{9,1}$ lasing modes. Even mode (a), (c): $k a=4.6981, \gamma=1.4901 * 10^{-2}, D=5.8$. Odd mode (b), (d): $k a=4.6973, \quad \gamma=1.4396 * 10^{-2}, D=4.94$. Limacon normalized deformation parameter $\delta=0.5, \alpha=2.63, N=40$.

Here, one should be reminded that in the circular microlaser, these modes are degenerate and the corresponding LEP eigenvalues are $k a=4.97799$ for the normalized lasing frequency and $\gamma=7.2896 \cdot 10^{-5}$ for the threshold material gain. In the limacon-deformed cavity, the sister modes of the same doublet are closely spaced on the plane $(k a, \gamma)$. For example, the differences in the frequencies and thresholds of the modes $\mathrm{WGH}_{9,1}$ appear only in the fourth digits (exact values are given in the caption to Fig. 2). As one can see, the circle deformation with $\delta=0.5$ leads to the growth of the threshold in 2000 times. 
Directionality of modal emission is quantified by the value of directivity,

$$
D=\frac{2 \pi}{P}\left|\Phi\left(\varphi_{\max }\right)\right|^{2}, \quad P=\int_{0}^{2 \pi}|\Phi(\varphi)|^{2} d \varphi,
$$

where $\varphi_{\max }$ is the angle of the main beam radiation in the halfspace $0 \leq \varphi \leq \pi, \Phi(\varphi)$ is far-field emission pattern, and $P$ is, within a constant, the total power radiated by a lasing mode.

The shapes of the near- and far-field radiation patterns of $\mathrm{WGH}_{9,1}$ doublets are shown in Fig. 2 for the maximum value of $\delta$ of the studied range, i.e. $\delta=0.5$.

As visible, the directivity can increase to more than twice larger value than for the unperturbed WG mode in a circle that equals 2. Note that the even-type mode of this doublet has a higher directivity than the sister mode of the odd type because the latter cannot have a single main beam. Instead, it always has a zero field emitted along the symmetry axis and thus radiates with minimum two identical main beams (Fig. 2 (d)).

\section{CONCLUSIONS}

We have presented preliminary results of the LEP-based numerical analysis of the lasing modes in the 2-D model of a limacon microcavity laser. This shape is attractive as it only slightly differs from the circle and has only single symmetry line. This combination of features enables one to keep relatively low thresholds of the perturbed WG modes and simultaneously improve their directivities of the light emission, especially for the modes of the even symmetry class. As one can see, even small deformations of this sort can lead to considerable changes of the emission patterns and provide attractively large directivities.

\section{ACKNOWLEDGMENTS}

This work has been partially supported by the National Academy of Sciences of Ukraine via the Target Program "Nanotechnologies and Nanomaterials" and the Ministry of Foreign and European Affairs, France and the State Agency for Science, Ukraine via the joint project "Dnipro."

\section{REFERENCES}

[1] J. Wiersig, M. Hentschel, "Combining directional light output and ultralow loss in deformed microdisks", Physical Review Lett., vol. 100, pp. 033901, 2008.

[2] Q. Song, W. Fang, B. Liu, S.-T. Ho, G. S. Solomon, H. Cao, "Chaotic microcavity laser with high quality factor and unidirectional output," Phys. Rev. A, vol. 80, no 4, pp. 1807-1811, 2009.

[3] Q.-J. Wang, C. Yan, L. Diehl, M. Hentschel, J. Wiersig, N. Yu, C. Pflugl, M. A Belkin, T. Edamura, M. Yamanishi, H. Kan, F. Capasso, "Deformed microcavity quantum cascade lasers with directional emission," New J. Physics, vol. 11, no 12, pp. 5018-5035, 2009.
[4] C.-H. Yi, M.-W. Kim, C.-M. Kim, "Lasing characteristics of a Limacon-shaped microcavity laser,” Appl. Phys. Lett., vol. 95, no 14, pp. 1107-1109, 2009.

[5] E.I. Smotrova, A.I. Nosich, T. M. Benson, P. Sewell, "Cold-cavity thresholds of microdisks with uniform and non-uniform gain: quasi-3D modelling with accurate 2D analysis,” IEEE J. Selected Topics Quant. Electron., vol. 11, no 5, pp. 1135-1142, 2005.

[6] E.I. Smotrova, V. O. Byelobrov, T. M. Benson, P. Sewell, J. Ctyroky, A. I. Nosich, "Optical theorem helps understand thresholds of lasing in microcavities with active regions," IEEE J. Quantum Electronics, vol. 47, no 1, pp. 20-30, 2011.

[7] L. Wang, J.A. Cox, A. Friedman, "Modal analysis of homogenious optical waveguides by the boundary integral formulation and the Nyström method," J. Opt. Soc. Am. A, vol. 15, no. 1, pp.92-100, 1998.

[8] D. Colton, R. Kress, Inverse Acoustic and Electromagnetic Scattering Theory, Springer, Berlin, 1998.

[9] J. Tsalamengas, "Exponentially converging Nystrom methods applied to the integral/integro-differential equations of oblique scattering hybrid wave propagation in presence of composite cylinders of arbitrary cross section,” IEEE Trans. Antennas Propag., vol. 55, no 11, pp. 3239-3250, 2007.

[10] E.I. Smotrova, T. M. Benson, P. Sewell, J. Ctyroky, R. Sauleau, A. I. Nosich, "Optical fields of the lowest modes in a uniformly active thin sub-wavelength spiral microcavity," Optics Letters, vol. 34, no 24, pp. 37733775, 2009.

[11]E.I. Smotrova, A. I. Nosich, "Directional light emission from a kite-shaped microcavity laser," Proc. Int. Conf. Transparent Optical Networks (ICTON-11), Stockholm, 2011, We.A4.4. 